\title{
Exponential Stability for a Class of Switched Nonlinear Systems with Mixed Time-Varying Delays via an Average Dwell-Time Method
}

\author{
N. Yotha, ${ }^{1,2}$ T. Botmart, ${ }^{2,3}$ and T. Mouktonglang1, \\ ${ }^{1}$ Department of Mathematics, Chiang Mai University, Chiang Mai 50200, Thailand \\ ${ }^{2}$ Centre of Excellence in Mathematics, CHE, Si Ayutthaya Road, Bangkok 10400, Thailand \\ ${ }^{3}$ Department of Mathematics, Srinakharinwirot University, Bangkok 10110, Thailand
}

Correspondence should be addressed to T. Botmart, thongchaib@swu.ac.th

Received 1 June 2012; Accepted 26 August 2012

Academic Editors: G. Mantica and M. Musso

Copyright (C) 2012 N. Yotha et al. This is an open access article distributed under the Creative Commons Attribution License, which permits unrestricted use, distribution, and reproduction in any medium, provided the original work is properly cited.

The problem of exponential stability for a class of switched nonlinear systems with discrete and distributed time-varying delays is studied. The constraint on the derivative of the time-varying delay is not required which allows the time delay to be a fast time-varying function. We study the stability properties of switched nonlinear systems consisting of both stable and unstable subsystems. Average dwell-time approached and improved piecewise Lyapunov functional combined with Leibniz-Newton are formulated. New delay-dependent sufficient conditions for the exponential stabilization of the switched systems are first established in terms of LMIs. A numerical example is also given to illustrate the effectiveness of the proposed method.

\section{Introduction}

The switched systems are an important class of hybrid systems. They are described by a family of continuous or discrete-time subsystems and a rule that orchestrates the switching between the subsystems. Recently, switched systems have attracted much attention due to the widespread application in control, chemical engineering processing [1], communication networks, traffic control [2,3], and control of manufacturing systems [4-6]. A switched nonlinear system with time delay is called switched nonlinear delay system, where delay may be contained in the system state, control input, or switching signals. In [7-9], some stability properties of switched linear delay systems composed of both stable and unstable subsystems have been studied by using an average dwell-time approach and piecewise 
Lyapunov functions. It is shown that when the average dwell time is sufficiently large and the total activation time of the unstable subsystems is relatively small compared with that of the Hurwitz stable subsystems, global exponential stability is guaranteed. The concept of dwell time was extended to average dwell time by Hespanha and Morse [10] with switching among stable subsystems. Furthermore, [11] generalized the results to the case where stable and unstable subsystems coexist.

The stability analysis of nonlinear time-delay systems has received increasing attention. Time-delay systems are frequently encountered in various areas such as chemical engineering systems, biological modeling, and economics. The stability analysis for nonlinear time-delay systems has been investigated extensively. Various approaches to such problems have been proposed, see [12-14] and the references therein. It is well known that the existences of time delay in a system may cause instability and oscillations system. Thus, the stability analysis of nonlinear time-delay systems has received considerable attention for the last few decades, see $[15,16]$.

Recently, the exponential stability problems for time-delay systems have been studied by many researchers, see for examples [17-21]. The problems have been dealt with for various control areas such as exponential stabilization for linear time-delay systems [22], exponential stabilization for uncertain time-delay systems [21, 23]. Hien and Phat [23] presented exponential stability and stabilization conditions for a class of uncertain linear system with time-varying delay, based on an improved Lyapunov-Krasovskii functional combined with Leibniz-Newton formula. The robust stability conditions are derived in terms of LMIs, but the time-varying delays are required to be differentiable and the lower bound is restricted to zero. However, in most cases, these conditions are difficult to satisfy. Therefore, in this paper, we will employ some new techniques so that the above conditions can be removed.

Stability analysis of nonlinear systems with distributed delays is of both practical and theoretical importance. For some systems, delay phenomena may not be simply considered as delays in the velocity terms and/or discrete delays in the states. Therefore, it is desirable to extend the system model to include distributed delays. Practical applications, modeled by systems with distributed delays, can be found in [24-26]. In [27], Gao et al. studied the problem of linear switching systems with discrete and continuous time delay. By constructing Lyapunov functional under a condition on the time delay, we show that it stabilizes the system for sufficiently small delays.

In this paper, the problem of exponential stability for a class of switched nonlinear systems with discrete and distributed time-varying delays is studied. The discrete time delay is a continuous function belonging to a given interval, which means that the lower and upper bounds for the time-varying delay are available. However, the delay function is not necessary to be differentiable. We study the stability properties of switched nonlinear systems consisting of both stable and unstable subsystems. Average dwell-time approached and improved piecewise Lyapunov functional combined with Leibniz-Newton's formula, and new delay-dependent sufficient conditions for the exponential stabilization of the switched systems are first established in terms of LMIs. A numerical example is also given to illustrate the effectiveness of the proposed method.

The rest of this paper is organized as follows. In Section 2, we give notations, definition, propositions, and lemma which will be used in the proof of the main results. Delay-dependent sufficient conditions for the exponential stability of switched nonlinear time-varying delay systems are presented in Section 3. A Numerical example illustrated the obtained results is given in Section 4. The paper ends with conclusions in Section 5 and cited references. 


\section{Preliminaries}

The following notation will be used in this paper. $\mathbb{R}^{+}$denotes the set of all real nonnegative numbers. $\mathbb{R}^{n}$ denotes the $n$-dimensional space and the vector norm $\|\cdot\| . M^{n \times r}$ denotes the space of all $(n \times r)$ matrices.

We also let $A^{T}$ denote the transpose of matrix $A: A$ is symmetric if $A=A^{T}: I$ denotes the identity matrix. $\lambda(A)$ denotes the set of all eigenvalues of $A . \lambda_{\max }(A)=\max \{\operatorname{Re} \lambda: \lambda \in$ $\lambda(A)\}: x_{t}:=\{x(t+s): s \in[-h, 0]\},\left\|x_{t}\right\|=\sup _{s \in[-h, 0]}\|x(t+s)\|: C\left([0, t], \mathbb{R}^{n}\right)$ denotes the set of all $\mathbb{R}^{n}$-valued continuous functions on $[0, t] . L_{2}\left([0, t], \mathbb{R}^{m}\right)$ denotes the set of all the $\mathbb{R}^{m}$-valued square integrable functions on $[0, t]$ : matrix $A$ is called semipositive definite $(A \geq 0)$ if $\langle A x, x\rangle \geq 0$, for all $x \in \mathbb{R}^{n}: A$ is positive definite $(A>0)$ if $\langle A x, x\rangle>0$ for all $x \neq 0: A>B$ means $A-B>0$ : the symmetric term in a matrix is denoted by $*$.

Consider a nonlinear system with interval time-varying and distributed delays of the form

$$
\begin{aligned}
\dot{x}(t)= & A_{\sigma(t)} x(t)+D_{\sigma(t)} x\left(t-h_{\sigma(t)}(t)\right)+C_{\sigma(t)} \int_{t-k_{\sigma(t)}(t)}^{t} x(s) d s \\
& +E_{\sigma(t)} f_{\sigma(t)}\left(t, x(t), x\left(t-h_{\sigma(t)}(t)\right), \int_{t-k_{\sigma(t)}(t)}^{t} x(s) d s\right), \\
x(t)= & \phi(t), \quad t \in[-\tau, 0], \tau=\max \left\{h_{2}, k\right\},
\end{aligned}
$$

where $x(t) \in \mathbb{R}^{n}$ is the state. $A_{\sigma(t)}, C_{\sigma(t)}, D_{\sigma(t)}$, and $E_{\sigma(t)}$ are given matrices of appropriate dimensions. $\sigma(t):[0,+\infty) \rightarrow M=\{1,2, \ldots, m\}$ is piecewise constant and left continuous and is called a switching signal and $\phi(t) \in C\left(\left[-h_{2}, 0\right], \mathbb{R}^{n}\right)$ is the initial function with the norm

$$
\|\phi\|=\sup _{s \in[-\tau, 0]} \sqrt{\|\phi(s)\|^{2}+\|\dot{\phi}(s)\|^{2}}
$$

The time delays $h(t)$ and $k(t)$ denoted the discrete time-varying delay and distributed timevarying delay, respectively. They are assumed to satisfy the following conditions:

$$
0 \leq h_{1 \sigma(t)} \leq h_{\sigma(t)}(t) \leq h_{2 \sigma(t)}, \quad 0 \leq k_{\sigma(t)}(t) \leq k_{\sigma(t)}
$$

The nonlinear perturbation satisfies the following growth condition:

$$
\begin{gathered}
\exists a_{i}, b_{i}, c_{i}>0, \quad\left\|f_{i}(t, x, y, z)\right\| \leq a_{i}\|x\|+b_{i}\|y\|+c_{i}\|z\|, \\
\forall(t, x, y, z) \in \mathbb{R}^{+} \times \mathbb{R}^{n} \times \mathbb{R}^{n} \times \mathbb{R}^{n}, \quad i \in M .
\end{gathered}
$$

We introduce the following technical well-known propositions, which will be used in the proof of our results.

Definition 2.1 (see [7]). For any switching signal $\sigma(t)$ and any $T_{2}>T_{1} \geq 0$, let $N_{\sigma}\left(T_{1}, T_{2}\right)$ denote the number of switching of $\sigma(t)$ over the interval $\left(T_{1}, T_{2}\right)$. For given $T_{a}>0, N_{0} \geq 0$, 
if the inequality $N_{\sigma}\left(T_{1}, T_{2}\right) \leq N_{0}+\left(\left(T_{2}-T_{1}\right) / T_{a}\right)$ holds, then $T_{a}$ is call average dwell time and $N_{0}$ is called chattering bound. As commonly used in the literature, for convenience, we choose $N_{0}=0$ in this paper.

Proposition 2.2 (Cauchy inequality). For any symmetric positive definite matrix $N \in M^{n \times n}$ and $x, y \in \mathbb{R}^{n}$ one has

$$
\pm 2 x^{T} y \leq x^{T} N x+y^{T} N^{-1} y
$$

Proposition 2.3 (see [28]). For any symmetric positive definite matrix $M>0$, scalar $\gamma>0$ and vector function $\omega:[0, \gamma] \rightarrow \mathbb{R}^{n}$ such that the integrations concerned are well defined, the following inequality holds:

$$
\left(\int_{0}^{\gamma} \omega(s) d s\right)^{T} M\left(\int_{0}^{\gamma} \omega(s) d s\right) \leq r\left(\int_{0}^{\gamma} \omega^{T}(s) M \omega(s) d s\right)
$$

Proposition 2.4 (see [28] (Schur complement lemma)). Given constant symmetric matrices $X, Y, Z$ with appropriate dimensions satisfying $X=X^{T}, Y=Y^{T}>0$. Then $X+Z^{T} Y^{-1} Z<0$ if and only if

$$
\left(\begin{array}{cc}
X & Z^{T} \\
Z & -Y
\end{array}\right)<0 \quad \text { or } \quad\left(\begin{array}{cc}
-Y & Z \\
Z^{T} & X
\end{array}\right)<0
$$

\section{Main Results}

\subsection{Stability of Switched Nonlinear Time-Varying Delay Systems}

First, we present a delay-dependent exponential stabilizability analysis conditions for the given nonlinear time-varying delay systems (2.1). We consider the case when the stable and unstable subsystems coexist. We assume that the subsystem $i(1 \leq i \leq k)$ is stable, where the positive integer $k$ satisfies $1 \leq k \leq m$ and the subsystem $j(k+1 \leq j \leq m)$ is unstable. Let us set $\eta_{i}=\left(h_{2 i}-h_{1 i}\right), i=1,2, \ldots, m$.

For the system (2.1), given $\alpha>0$, the following lemma provides a change estimation of Lyapunov-Krasovskii functional candidate:

$$
V_{i}(x(t))=V_{i 1}+V_{i 2}+V_{i 3}+V_{i 4}+V_{i 5}+V_{i 6}+V_{i 7},
$$

where

$$
\begin{aligned}
& V_{i 1}=x^{T}(t) Y_{i} x(t), \\
& V_{i 2}=\int_{t-h_{1 i}}^{t} e^{\alpha(s-t)} x^{T}(s) Y_{i} Q_{i} Y_{i} x(s) d s, \\
& V_{i 3}=\int_{t-h_{2 i}}^{t} e^{\alpha(s-t)} x^{T}(s) Y_{i} Q_{i} Y_{i} x(s) d s,
\end{aligned}
$$




$$
\begin{aligned}
& V_{i 4}=h_{1 i} \int_{-h_{1 i}}^{0} \int_{t+s}^{t} e^{\alpha(\tau-t)} \dot{x}^{T}(\tau) Y_{i} R_{i} Y_{i} \dot{x}(\tau) d \tau d s, \\
& V_{i 5}=h_{2 i} \int_{-h_{2 i}}^{0} \int_{t+s}^{t} e^{\alpha(\tau-t)} \dot{x}^{T}(\tau) Y_{i} R_{i} Y_{i} \dot{x}(\tau) d \tau d s, \\
& V_{i 6}=\eta_{i} \int_{-h_{2 i}}^{-h_{1 i}} \int_{t+s}^{t} e^{\alpha(\tau-t)} \dot{x}^{T}(\tau) Y_{i} U_{i} Y_{i} \dot{x}(\tau) d \tau d s, \\
& V_{i 7}=\int_{k_{i}}^{0} \int_{t+s}^{t} e^{\alpha(\tau-t)} x^{T}(\tau) Y_{i} S_{i} Y_{i} x(\tau) d \tau d s .
\end{aligned}
$$

Lemma 3.1. For a given constant $\alpha>0$, suppose (2.3) holds. If there exist symmetric positive definite matrices $P_{i}, Q_{i}, R_{i}, U_{i}, S_{i}$ such that the following LMI holds:

$$
\begin{aligned}
& \Sigma_{1 i}=W_{i}-\left[\begin{array}{llllllll}
0 & 0 & 0 & -I & I & 0 & 0 & 0
\end{array}\right]^{T} \\
& \times e^{-\alpha h_{2 i}} U_{i}\left[\begin{array}{llllllll}
0 & 0 & 0 & -I & I & 0 & 0 & 0
\end{array}\right]<0, \\
& \Sigma_{2 i}=W_{i}-\left[\begin{array}{llllllll}
0 & 0 & I & 0 & -I & 0 & 0 & 0
\end{array}\right]^{T} \\
& \times e^{-\alpha h_{2 i}} U_{i}\left[\begin{array}{llllllll}
0 & 0 & I & 0 & -I & 0 & 0 & 0
\end{array}\right]<0, \\
& \Sigma_{3 i}=\left[\begin{array}{cccc}
\Psi_{i} & k_{i} C_{i} P_{i} & 2 a_{i} P_{i} E_{i}^{T} & k_{i} c_{i}^{2} E_{i} P_{i} \\
* & \frac{-k_{i} e^{-\alpha k_{i}} S_{i}}{4} & 0 & 0 \\
* & * & -2 a_{i} I & 0 \\
* & * & * & \frac{-k_{i} c_{i}^{2} e^{-\alpha k_{i}} S_{i}}{4}
\end{array}\right]<0, \\
& W_{i}=\left[\begin{array}{cccccccc}
W_{11 i} & W_{12 i} & W_{13 i} & W_{14 i} & W_{15 i} & 0 & 0 & 0 \\
* & W_{22 i} & 0 & 0 & W_{25 i} & k_{i} C_{i} P_{i} & k_{i} c_{i}^{2} E_{i} P_{i} & 0 \\
* & * & W_{33 i} & 0 & W_{35 i} & 0 & 0 & 0 \\
* & * & * & W_{44 i} & W_{45 i} & 0 & 0 & 0 \\
* & * & * & * & W_{55 i} & 0 & 0 & 2 b_{i} P_{i} E_{i}^{T} \\
* & * & * & * & * & W_{66 i} & 0 & 0 \\
* & * & * & * & * & * & W_{77 i} & 0 \\
* & * & * & * & * & * & * & -2 b_{i} I
\end{array}\right], \\
& i=1,2,3, \ldots, k \text {, }
\end{aligned}
$$

where

$$
\begin{aligned}
\Psi_{i} & =-0.1 e^{-\alpha h_{1 i}} R_{i}-0.1 e^{-\alpha h_{2 i}} R_{i}, \\
W_{11 i} & =\left[A_{i}+\alpha I\right] P_{i}+P_{i}\left[A_{i}+\alpha I\right]^{T}-0.9\left(e^{-\alpha h_{1 i}}+e^{-\alpha h_{2 i}}\right) R_{i}+2 Q_{i}+k_{i} S_{i}+\left(a_{i}+b_{i}\right) I, \\
W_{12 i} & =P_{i} A_{i}^{T},
\end{aligned}
$$




$$
\begin{aligned}
& W_{13 i}=e^{-\alpha h_{1 i}} R_{i}, \\
& W_{14 i}=e^{-\alpha h_{2 i}} R_{i}, \\
& W_{15 i}=D_{i} P_{i}, \\
& W_{22 i}=\left(h_{1 i}^{2}+h_{2 i}^{2}\right) R_{i}+\eta_{i}^{2} U_{i}-2 P_{i}+\left(a_{i}+b_{i}\right) I, \\
& W_{25 i}=D_{i} P_{i}, \\
& W_{33 i}=-e^{-\alpha h_{1 i}}\left(Q_{i}+R_{i}\right)-e^{-\alpha h_{2 i}} U_{i}, \\
& W_{35 i}=e^{-\alpha h_{2 i}} U_{i}, \\
& W_{44 i}=-e^{-\alpha h_{2 i}}\left(Q_{i}+R_{i}+U_{i}\right), \\
& W_{45 i}=e^{-\alpha h_{2 i}} U_{i}, \\
& W_{55 i}=-2 e^{-\alpha h_{2 i}} U_{i}, \\
& W_{66 i}=-\frac{k_{i} e^{-\alpha k_{i}} S_{i}}{4}, \\
& W_{77 i}=-\frac{k_{i} c_{i}^{2} e^{-\alpha k_{i}} S_{i}}{4} .
\end{aligned}
$$

Then, along the trajectory of system (2.1), one has

$$
V_{i}(x(t)) \leq e^{-\alpha\left(t-t_{0}\right)} V_{i}\left(x\left(t_{0}\right)\right), \quad t \geq t_{0} \geq 0 .
$$

Proof. Let $Y_{i}=P_{i}^{-1}, y(t)=Y_{i} x(t)$. We consider the following Lyapunov-Krasovskii functional (3.1). By taking the derivative of Lyapunov-Krasovskii functional candidate (3.1) along the trajectory of the system (2.1) we have

$$
\begin{aligned}
\dot{V}_{i 1}= & y^{T}(t)\left[P_{i} A_{i}^{T}+A_{i} P_{i}\right] y(t)+2 y^{T}(t) D_{i} P_{i} y\left(t-h_{i}(t)\right)+2 y^{T}(t) C_{i} P_{i} \int_{t-k_{i}(t)}^{t} y(s) d s \\
& +2 y^{T}(t) E_{i} f_{i}\left(t, x(t), x\left(t-h_{i}(t)\right), \int_{t-k_{i}(t)}^{t} x(s) d s\right), \\
\dot{V}_{i 2}= & y^{T}(t) Q_{i} y(t)-e^{-\alpha h_{1 i}} y^{T}\left(t-h_{1 i}\right) Q_{i} y\left(t-h_{1 i}\right)-\alpha V_{i 2}, \\
\dot{V}_{i 3}= & y^{T}(t) Q_{i} y(t)-e^{-\alpha h_{2 i}} y^{T}\left(t-h_{2 i}\right) Q_{i} y\left(t-h_{2 i}\right)-\alpha V_{i 3}, \\
\dot{V}_{i 4} \leq & h_{1 i}^{2} \dot{y}^{T}(t) R_{i} \dot{y}(t)-h_{1 i} e^{-\alpha h_{1 i}} \int_{t-h_{1 i}}^{t} \dot{y}^{T}(s) R_{i} \dot{y}(s) d s-\alpha V_{i 4},
\end{aligned}
$$




$$
\begin{aligned}
& \dot{V}_{i 5} \leq h_{2 i}^{2} \dot{y}^{T}(t) R_{i} \dot{y}(t)-h_{2 i} e^{-\alpha h_{2 i}} \int_{t-h_{2 i}}^{t} \dot{y}^{T}(s) R_{i} \dot{y}(s) d s-\alpha V_{i 5}, \\
& \dot{V}_{i 6} \leq \eta_{i}^{2} \dot{y}^{T}(t) U_{i} \dot{y}(t)-\eta_{i} e^{-\alpha h_{2 i}} \int_{t-h_{2 i}}^{t-h_{1 i}} \dot{y}^{T}(s) U_{i} \dot{y}(s) d s-\alpha V_{i 6}, \\
& \dot{V}_{i 7} \leq k_{i} y^{T}(t) S_{i} y(t)-e^{-\alpha k_{i}} \int_{t-k_{i}(t)}^{t} y^{T}(s) S_{i} y(s) d s-\alpha V_{i 7} .
\end{aligned}
$$

Applying Proposition 2.3 and the Leibniz-Newton formula, we have

$$
\begin{aligned}
-h_{1} \int_{t-h_{1 i}}^{t} \dot{y}^{T}(s) R_{i} \dot{y}(s) d s & \leq-\left[\int_{t-h_{1 i}}^{t} \dot{y}(s) d s\right]^{T} R_{i}\left[\int_{t-h_{1 i}}^{t} \dot{y}(s) d s\right] \\
& \leq-\left[y(t)-y\left(t-h_{1 i}\right)\right]^{T} R_{i}\left[y(t)-y\left(t-h_{1 i}\right)\right] \\
& =-y^{T}(t) R_{i} y(t)+2 y^{T}(t) R_{i} y\left(t-h_{1 i}\right)-y^{T}\left(t-h_{1 i}\right) R_{i} y\left(t-h_{1 i}\right), \\
-h_{2} \int_{t-h_{2 i}}^{t} \dot{y}^{T}(s) R_{i} \dot{y}(s) d s & \leq-\left[\int_{t-h_{2 i}}^{t} \dot{y}(s) d s\right]^{T} R_{i}\left[\int_{t-h_{2 i}}^{t} \dot{y}(s) d s\right] \\
& \leq-\left[y(t)-y\left(t-h_{2 i}\right)\right]^{T} R_{i}\left[y(t)-y\left(t-h_{2 i}\right)\right] \\
& =-y^{T}(t) R_{i} y(t)+2 y^{T}(t) R_{i} y\left(t-h_{2 i}\right)-y^{T}\left(t-h_{2 i}\right) R_{i} y\left(t-h_{2 i}\right) .
\end{aligned}
$$

Note that

$$
\begin{aligned}
-\left(h_{2 i}-h_{1 i}\right) \int_{t-h_{2 i}}^{t-h_{1 i}} \dot{y}^{T}(s) U_{i} \dot{y}(s) d s= & -\left(h_{2 i}-h_{1 i}\right) \int_{t-h_{2 i}}^{t-h_{i}(t)} \dot{y}^{T}(s) U_{i} \dot{y}(s) d s \\
& -\left(h_{2 i}-h_{1 i}\right) \int_{t-h_{i}(t)}^{t-h_{1 i}} \dot{y}^{T}(s) U_{i} \dot{y}(s) d s \\
= & -\left(h_{2 i}-h_{i}(t)\right) \int_{t-h_{2 i}}^{t-h_{i}(t)} \dot{y}^{T}(s) U_{i} \dot{y}(s) d s \\
& -\left(h_{i}(t)-h_{1 i}\right) \int_{t-h_{2 i}}^{t-h_{i}(t)} \dot{y}^{T}(s) U_{i} \dot{y}(s) d s \\
& -\left(h_{i}(t)-h_{1 i}\right) \int_{t-h_{i}(t)}^{t-h_{1 i}} \dot{y}^{T}(s) U_{i} \dot{y}(s) d s \\
& -\left(h_{2 i}-h_{i}(t)\right) \int_{t-h_{i}(t)}^{t-h_{1 i}} \dot{y}^{T}(s) U_{i} \dot{y}(s) d s .
\end{aligned}
$$


Using Proposition 2.3 gives

$$
\begin{aligned}
-\left(h_{2 i}-h_{i}(t)\right) \int_{t-h_{2 i}}^{t-h_{i}(t)} \dot{y}^{T}(s) U_{i} \dot{y}(s) d s & \leq-\left[\int_{t-h_{2 i}}^{t-h_{i}(t)} \dot{y}(s) d s\right]^{T} U_{i}\left[\int_{t-h_{2 i}}^{t-h_{i}(t)} \dot{y}(s) d s\right] \\
& \leq-\left[y\left(t-h_{i}(t)\right)-y\left(t-h_{2 i}\right)\right]^{T} U_{i}\left[y\left(t-h_{i}(t)\right)-y\left(t-h_{2 i}\right)\right], \\
-\left(h_{i}(t)-h_{1 i}\right) \int_{t-h_{i}(t)}^{t-h_{1 i}} \dot{y}^{T}(s) U_{i} \dot{y}(s) d s & \leq-\left[\int_{t-h_{i}(t)}^{t-h_{1 i}} \dot{y}(s) d s\right]^{T} U_{i}\left[\int_{t-h_{i}(t)}^{t-h_{1 i}} \dot{y}(s) d s\right] \\
& \leq-\left[y\left(t-h_{1 i}\right)-y\left(t-h_{i}(t)\right)\right]^{T} U_{i}\left[y\left(t-h_{1 i}\right)-y\left(t-h_{i}(t)\right)\right] .
\end{aligned}
$$

Let $\beta_{i}=\left(\left(h_{2 i}-h_{i}(t)\right) /\left(h_{2 i}-h_{1 i}\right)\right) \leq 1$. Then

$$
\begin{aligned}
-\left(h_{2 i}-h_{i}(t)\right) \int_{t-h_{i}(t)}^{t-h_{1}} \dot{y}^{T}(s) U_{i} \dot{y}(s) d s= & -\beta_{i} \int_{t-h_{i}(t)}^{t-h_{1}}\left(h_{2 i}-h_{1 i}\right) \dot{y}^{T}(s) U_{i} \dot{y}(s) d s \\
\leq & -\beta_{i} \int_{t-h_{i}(t)}^{t-h_{1}}\left(h_{i}(t)-h_{1 i}\right) \dot{y}^{T}(s) U_{i} \dot{y}(s) d s \\
\leq & -\beta_{i}\left[y\left(t-h_{1 i}\right)-y\left(t-h_{i}(t)\right)\right]^{T} U_{i}\left[y\left(t-h_{1 i}\right)-y\left(t-h_{i}(t)\right)\right] \\
-\left(h_{i}(t)-h_{1 i}\right) \int_{t-h_{2 i}}^{t-h_{i}(t)} \dot{y}^{T}(s) U_{i} \dot{y}(s) d s= & -\left(1-\beta_{i}\right) \int_{t-h_{2 i}}^{t-h_{i}(t)}\left(h_{2 i}-h_{1 i}\right) \dot{y}^{T}(s) U_{i} \dot{y}(s) d s \\
\leq & -\left(1-\beta_{i}\right) \int_{t-h_{2 i}}^{t-h_{i}(t)}\left(h_{2 i}-h(t)\right) \dot{y}^{T}(s) U_{i} \dot{y}(s) d s \\
\leq & -\left(1-\beta_{i}\right)\left[y\left(t-h_{i}(t)\right)-y\left(t-h_{2 i}\right)\right]^{T} \\
& \times U_{i}\left[y\left(t-h_{i}(t)\right)-y\left(t-h_{2 i}\right)\right] .
\end{aligned}
$$

Therefore, from (3.12) and (3.13), we obtain

$$
\begin{aligned}
-\left(h_{2 i}-h_{1 i}\right) \int_{t-h_{2 i}}^{t-h_{1 i}} \dot{y}^{T}(s) U_{i} \dot{y}(s) d s \\
\quad \leq-\left[y\left(t-h_{i}(t)\right)-y\left(t-h_{2 i}\right)\right]^{T} U_{i}\left[y\left(t-h_{i}(t)\right)-y\left(t-h_{2 i}\right)\right] \\
-\left[y\left(t-h_{1 i}\right)-y\left(t-h_{i}(t)\right)\right]^{T} U_{i}\left[y\left(t-h_{1 i}\right)-y\left(t-h_{i}(t)\right)\right] \\
-\beta_{i}\left[y\left(t-h_{1 i}\right)-y\left(t-h_{i}(t)\right)\right]^{T} U_{i}\left[y\left(t-h_{1 i}\right)-y\left(t-h_{i}(t)\right)\right] \\
-\left(1-\beta_{i}\right)\left[y\left(t-h_{i}(t)\right)-y\left(t-h_{2 i}\right)\right]^{T} U_{i}\left[y\left(t-h_{i}(t)\right)-y\left(t-h_{2 i}\right)\right] .
\end{aligned}
$$


By using the following identity relation

$$
\begin{gathered}
P_{i} \dot{y}(t)-A_{i} P_{i} y(t)-D_{i} P_{i} y\left(t-h_{i}(t)\right)-C_{i} P_{i} \int_{t-k_{i}(t)}^{t} y(s) d s \\
-E_{i} f_{i}\left(t, x(t), x\left(t-h_{i}(t)\right), \int_{t-k_{i}(t)}^{t} x(s) d s\right)=0,
\end{gathered}
$$

we have

$$
\begin{aligned}
& -2 \dot{y}^{T}(t) P_{i} \dot{y}(t)+2 \dot{y}^{T}(t) A_{i} P_{i} y(t)+2 \dot{y}^{T}(t) D_{i} P_{i} y\left(t-h_{i}(t)\right)+2 \dot{y}^{T}(t) C_{i} P_{i} \int_{t-k_{i}(t)}^{t} y(s) d s \\
& +2 \dot{y}^{T}(t) E_{i} f_{i}\left(t, x(t), x\left(t-h_{i}(t)\right), \int_{t-k_{i}(t)}^{t} x(s) d s\right)=0 .
\end{aligned}
$$

Applying Propositions 2.2 and 2.3 gives

$$
\begin{aligned}
2 y^{T}(t) C_{i} P_{i} \int_{t-k_{i}(t)}^{t} y(s) d s \leq & 4 k_{i} e^{\alpha k_{i}} y^{T}(t) C_{i} P_{i} S_{i}^{-1} P_{i} C_{i}^{T} y(t) \\
& +\frac{1}{4 k_{i}} e^{-\alpha k_{i}}\left(\int_{t-k_{i}(t)}^{t} y(s) d s\right)^{T} S_{i}\left(\int_{t-k_{i}(\mathrm{t})}^{t} y(s) d s\right) \\
\leq & 4 k_{i} e^{\alpha k_{i}} y^{T}(t) C_{i} P_{i} S_{i}^{-1} P_{i} C_{i}^{T} y(t) \\
& +\frac{1}{4} e^{-\alpha k_{i}} \int_{t-k_{i}(t)}^{t} y^{T}(s) S_{i} y(s) d s, \\
2 \dot{y}^{T}(t) C_{i} P_{i} \int_{t-k_{i}(t)}^{t} y(s) d s \leq & 4 k_{i} e^{\alpha k_{i}} \dot{y}^{T}(t) C_{i} P_{i} S_{i}^{-1} P_{i} C_{i}^{T} \dot{y}(t) \\
& +\frac{1}{4 k_{i}} e^{-\alpha k_{i}}\left(\int_{t-k_{i}(t)}^{t} y(s) d s\right)^{T} S_{i}\left(\int_{t-k_{i}(t)}^{t} y(s) d s\right) \\
\leq & 4 k_{i} e^{\alpha k_{i}} \dot{y}^{T}(t) C_{i} P_{i} S_{i}^{-1} P_{i} C_{i}^{T} \dot{y}(t) \\
& +\frac{1}{4} e^{-\alpha k_{i}} \int_{t-k_{i}(t)}^{t} y^{T}(s) S_{i} y(s) d s .
\end{aligned}
$$


By using Proposition 2.2 and condition (2.4), we have

$$
\begin{aligned}
& 2 y^{T}(t) E_{i} f_{i}\left(t, x(t), x(t-h(t)), \int_{t-k(t)}^{t} x(s) d s\right) \\
& \leq 2\|y(t)\| E_{i}\left\|f_{i}\left(t, x(t), x(t-h(t)), \int_{t-k(t)}^{t} x(s) d s\right)\right\| \\
& \leq 2\|y(t)\| E_{i}\left(a_{i}\|x\|+b_{i}\left\|x\left(t-h_{i}(t)\right)\right\|+c_{i}\left\|\int_{t-k_{i}(t)}^{t} x(s) d s\right\|\right) \\
& =2 a_{i}\|y(t)\|\left\|E_{i} P_{i} y(t)\right\|+2 b_{i}\|y(t)\|\left\|E_{i} P_{i} y\left(t-h_{i}(t)\right)\right\|+2 c_{i}\|y(t)\|\left\|E_{i} P_{i} \int_{t-k_{i}(t)}^{t} y(s) d s\right\| \\
& \leq\left(a_{i}+b_{i}\right) y^{T}(t) y(t)+a_{i} y^{T}(t) P_{i} E_{i}^{T} E_{i} P_{i} y(t)+b_{i} y^{T}\left(t-h_{i}(t)\right) P_{i} E_{i}^{T} E_{i} P_{i} y(t-h(t)) \\
& \quad+4 k_{i} c_{i}^{2} e^{\alpha k_{i}} y^{T}(t) E_{i} P_{i} S_{i}^{-1} P_{i} E_{i}^{T} y(t)+\frac{1}{4} e^{-\alpha k_{i}} \int_{t-k_{i}(t)}^{t} y^{T}(s) S_{i} y(s) d s, \\
& 2 \dot{y}^{T}(t) E_{i} f_{i}\left(t, x(t), x(t-h(t)), \int_{t-k(t)}^{t} x(s) d s\right) \\
& \leq\left(a_{i}+b_{i}\right) \dot{y}^{T}(t) \dot{y}(t)+a_{i} y^{T}(t) P_{i} E_{i}^{T} E_{i} P_{i} y(t)+b_{i} y^{T}\left(t-h_{i}(t)\right) P_{i} E_{i}^{T} E_{i} P_{i} y\left(t-h_{i}(t)\right) \\
& \quad+4 k_{i} c_{i}^{2} e^{\alpha k_{i}} \dot{y}^{T}(t) E_{i} P_{i} S_{i}^{-1} P_{i} E_{i}^{T} \dot{y}(t)+\frac{1}{4} e^{-\alpha k_{i}} \int_{t-k_{i}(t)}^{t} y^{T}(s) S_{i} y(s) d s .
\end{aligned}
$$

Hence, according to (3.10)-(3.18), we have

$$
\begin{aligned}
\dot{V}_{i}(x(t))+\alpha V_{i}(x(t)) \leq & \zeta^{T}(t) W_{i} \zeta(t) \\
& -\beta_{i}\left[y\left(t-h_{1 i}\right)-y\left(t-h_{i}(t)\right)\right]^{T} e^{-\alpha h_{2 i}} U_{i}\left[y\left(t-h_{1 i}\right)-y\left(t-h_{i}(t)\right)\right] \\
& -\left(1-\beta_{i}\right)\left[y\left(t-h_{i}(t)\right)-y\left(t-h_{2 i}\right)\right]^{T} e^{-\alpha h_{2 i}} U_{i}\left[y\left(t-h_{i}(t)\right)-y\left(t-h_{2 i}\right)\right] \\
& =\zeta^{T}(t)\left[\left(1-\beta_{i}\right) \Sigma_{1 i}+\beta_{i} \Sigma_{2 i}\right] \zeta(t)+y^{T}(t) \Sigma_{i} y(t)
\end{aligned}
$$

where $\Sigma_{1 i}$ and $\Sigma_{2 i}$ are defined in (3.3), (3.4), respectively, and

$$
\begin{gathered}
\Sigma_{i}=\Psi_{i}+4 k_{i} e^{\alpha k_{i}} C_{i} P_{i} S_{i}^{-1} P_{i} C_{i}^{T}+2 a_{i} P_{i} E_{i}^{T} E_{i} P_{i}+4 k_{i} c_{i}^{2} e^{\alpha k_{i}} E_{i} P_{i} S_{i}^{-1} P_{i} E_{i}^{T}, \\
\zeta(t)=\left[y(t), \dot{y}(t), y\left(t-h_{1 i}\right), y\left(t-h_{2 i}\right), y(t-h(t))\right] .
\end{gathered}
$$


Since $0 \leq \beta_{i} \leq 1,\left(1-\beta_{i}\right) \Sigma_{1 i}+\beta_{i} \Sigma_{2 i}$ is a convex combination of $\Sigma_{1 i}$ and $\Sigma_{2 i}$, therefore, (1$\left.\beta_{i}\right) \Sigma_{1 i}+\beta_{i} \Sigma_{2 i}<0$ is equivalent to $\Sigma_{1 i}<0$ and $\Sigma_{2 i}<0$. Applying Shur complement lemma, the inequalities $\Sigma_{i}$ is equivalent to $\Sigma_{3 i}<0$. Thus, it follows from (3.3)-(3.5) and (3.19), we obtain

$$
\dot{V}(x(t))+\alpha V(x(t)) \leq 0, \quad t \geq t_{0} \geq 0 .
$$

Thus, by the above differential inequality, we have

$$
V_{i}(x(t)) \leq e^{-\alpha\left(t-t_{0}\right)} V_{i}\left(x\left(t_{0}\right)\right), \quad t \geq t_{0} \geq 0 .
$$

For the system (2.1) and given $\beta>0$, the following lemma provides a change estimate of Lyapunov-Krasovskii functional candidate:

$$
V_{j}(x(t))=V_{j 1}+V_{j 2}+V_{j 3}+V_{j 4}+V_{j 5}+V_{j 6}+V_{j 7}
$$

where

$$
\begin{aligned}
& V_{j 1}=x^{T}(t) Y_{j} x(t), \\
& V_{j 2}=\int_{t-h_{1 j}}^{t} e^{\beta(t-s)} x^{T}(s) Y_{j} Q_{j} Y_{j} x(s) d s, \\
& V_{j 3}=\int_{t-h_{2 j}}^{t} e^{\beta(t-s)} x^{T}(s) Y_{j} Q_{j} Y_{j} x(s) d s, \\
& V_{j 4}=h_{1 i} \int_{-h_{1 j}}^{0} \int_{t+s}^{t} e^{\beta(t-\tau)} \dot{x}^{T}(\tau) Y_{j} R_{j} Y_{j} \dot{x}(\tau) d \tau d s, \\
& V_{j 5}=h_{2 j} \int_{-h_{2 j}}^{0} \int_{t+s}^{t} e^{\beta(t-\tau)} \dot{x}^{T}(\tau) Y_{j} R_{j} Y_{j} \dot{x}(\tau) d \tau d s, \\
& V_{j 6}=\eta_{j} \int_{-h_{2 j}}^{-h_{1 j}} \int_{t+s}^{t} e^{\beta(t-\tau)} \dot{x}^{T}(\tau) Y_{j} U_{j} Y_{j} \dot{x}(\tau) d \tau d s, \\
& V_{j 7}=\int_{k_{j}}^{0} \int_{t+s}^{t} e^{\beta(t-\tau)} x^{T}(\tau) Y_{j} S_{j} Y_{j} x(\tau) d \tau d s .
\end{aligned}
$$


Lemma 3.2. For a given constant $\beta>0$, suppose (2.3) holds. If there exist symmetric positive definite matrices $P_{j}, Q_{j}, R_{j}, U_{j}, S_{j}$ such that the following $L M I$ holds:

$$
\begin{aligned}
& \Sigma_{1 j}=W_{j}-\left[\begin{array}{llllllll}
0 & 0 & 0 & -I & I & 0 & 0 & 0
\end{array}\right]^{T} \\
& \times e^{\beta h_{2 j}} U_{j}\left[\begin{array}{llllllll}
0 & 0 & 0 & -I & I & 0 & 0 & 0
\end{array}\right]<0, \\
& \Sigma_{2 j}=W_{j}-\left[\begin{array}{llllllll}
0 & 0 & I & 0 & -I & 0 & 0 & 0
\end{array}\right]^{T} \\
& \times e^{\beta h_{2 j}} U_{j}\left[\begin{array}{llllllll}
0 & 0 & I & 0 & -I & 0 & 0 & 0
\end{array}\right]<0, \\
& \Sigma_{3 j}=\left[\begin{array}{cccc}
\Psi_{j} & k_{j} C_{j} P_{j} & 2 a_{j} P_{j} E_{j}^{T} & k_{j} c_{j}^{2} E_{j} P_{j} \\
* & \frac{-k_{j} e^{\beta k_{j}} S_{j}}{4} & 0 & 0 \\
* & * & -2 a_{j} I & 0 \\
* & * & * & \frac{-k_{j} c_{j}^{2} e^{\beta k_{j}} S_{j}}{4}
\end{array}\right]<0, \\
& W_{j}=\left[\begin{array}{cccccccc}
W_{11 j} & W_{12 j} & W_{13 j} & W_{14 j} & W_{15 j} & 0 & 0 & 0 \\
* & W_{22 j} & 0 & 0 & W_{25 j} & k_{j} C_{j} P_{j} & k_{j} c_{j}^{2} E_{j} P_{j} & 0 \\
* & * & W_{33 j} & 0 & W_{35 j} & 0 & 0 & 0 \\
* & * & * & W_{44 j} & W_{45 j} & 0 & 0 & 0 \\
* & * & * & * & W_{55 j} & 0 & 0 & 2 b_{j} P_{j} E_{j}^{T} \\
* & * & * & * & * & W_{66 j} & 0 & 0 \\
* & * & * & * & * & * & W_{77 j} & 0 \\
* & * & * & * & * & * & * & -2 b_{j} I
\end{array}\right],
\end{aligned}
$$

where

$$
\begin{aligned}
\Psi_{j} & =-0.1 e^{\beta h_{1 j}} R_{j}-0.1 e^{\beta h_{2 j}} R_{j}, \\
W_{11 j} & =\left[A_{j}-\beta I\right] P_{j}+P_{j}\left[A_{j}-\beta I\right]^{T}-0.9\left(e^{\beta h_{1 j}}+e^{\beta h_{2 j}}\right) R_{j}+2 Q_{j}+k_{j} S_{j}+\left(a_{j}+b_{j}\right) I, \\
W_{12 j} & =P_{j} A_{j}^{T}, \\
W_{13 j} & =e^{\beta h_{1 j}} R_{j}, \\
W_{14 j} & =e^{\beta h_{2 j}} R_{j} \\
W_{15 j} & =D_{j} P_{j} \\
W_{22 j} & =\left(h_{1 j}^{2}+h_{2 j}^{2}\right) R_{j}+\eta_{j}^{2} U_{j}-2 P_{j}+\left(a_{j}+b_{j}\right) I, \\
W_{25 j} & =D_{j} P_{j}, \\
W_{33 j} & =-e^{\beta h_{1 j}}\left(Q_{j}+R_{j}\right)-e^{\beta h_{2 j}} U_{j}, \\
W_{35 j} & =e^{\beta h_{2 j}} U_{j},
\end{aligned}
$$




$$
\begin{aligned}
& W_{44 j}=-e^{\beta h_{2 j}}\left(Q_{j}+R_{j}+U_{j}\right), \\
& W_{45 j}=e^{\beta h_{2 j}} U_{j}, \\
& W_{55 j}=-2 e^{\beta h_{2 j}} U_{j}, \\
& W_{66 j}=-\frac{k_{j} e^{\beta k_{j}} S_{j}}{4}, \\
& W_{77 j}=-\frac{k_{j} c_{j}^{2} e^{\beta k_{j}} S_{j}}{4} .
\end{aligned}
$$

Then, along the trajectory of system (2.1), one has

$$
V_{j}(x(t)) \leq e^{\beta\left(t-t_{0}\right)} V_{j}\left(x\left(t_{0}\right)\right), \quad t \geq t_{0} \geq 0 .
$$

Proof. By taking the derivative of Lyapunov-Krasovskii functional candidate (3.23) along the trajectory of the system (2.1), we are able to do similar estimation as we did for the Lemma 3.1. We have the following:

$$
\dot{V}_{j}(t)-\beta V_{j}(t) \leq 0, \quad t \geq t_{0} \geq 0 .
$$

Thus, by the above differential inequality, we have

$$
V_{j}(x(t)) \leq e^{\beta\left(t-t_{0}\right)} V_{j}\left(x\left(t_{0}\right)\right), \quad t \geq t_{0} \geq 0 .
$$

From Lemmas 3.1 and 3.2, it is easy to show the following properties of the Lyapunov functional candidate (3.1) and (3.23).

(i) There exist scalars $\varepsilon_{1}>0, \varepsilon_{2}>0$, such that

$$
\varepsilon_{1}\|x(t)\|^{2} \leq V_{\sigma(t)}(x(t)) \leq \varepsilon_{2}\left\|x\left(t_{0}\right)\right\|_{\mathrm{cl}^{\prime}}^{2} \quad \sigma(t) \in M .
$$

(ii) There exists a constant scalar $\mu \geq 1$ such that

$$
V_{i}(x(t)) \leq \mu V_{j}(x(t)), \quad i, j \in M .
$$

(iii) The Lyapunov functional candidate (3.1) and (3.23) whose derivative along the trajectory of the corresponding subnetwork satisfies

$$
V(x(t)) \leq \begin{cases}e^{-\alpha\left(t-t_{k}\right)} V_{i}\left(x\left(t_{k}\right)\right), & \text { if } i \in 1,2, \ldots, k, \text { for } t \in\left[t_{k}, t_{k+1}\right), \\ e^{\beta\left(t-t_{k}\right)} V_{j}\left(x\left(t_{k}\right)\right), & \text { if } j \in k+1, k+2, \ldots, m, \text { for } t \in\left[t_{k}, t_{k+1}\right) .\end{cases}
$$


Now, for any piecewise constant switching signal $\sigma(t)$ and any $0 \leq t_{0}<t$, we let $T^{-}\left(t_{0}, t\right)$ (resp., $T^{+}\left(t_{0}, t\right)$ ) denote the total activation time of the stable subsystem (resp., the ones of unstable subsystem) during $\left(t_{0}, t\right)$. Then, we choose a scalar $\alpha^{*} \in(0, \alpha)$ arbitrarily to propose the following switching law:

$(S 1)$ : determine the switching signal $\sigma(t)$ so that

$$
\inf _{t \geq t_{0}} \frac{T^{-}\left(t_{0}, t\right)}{T^{+}\left(t_{0}, t\right)} \geq \frac{\beta+\alpha^{*}}{\alpha-\alpha^{*}}
$$

holds on time interval $\left(t_{0}, t\right)$. Meanwhile, we choose $\alpha^{*}<\alpha$ as the average dwell-time scheme: for any $t>t_{0}$,

$$
N_{\sigma}\left(t_{0}, t\right) \leq N_{0}+\frac{t-t_{0}}{T_{a}}, \quad T_{a} \geq T_{a}^{*}=\frac{\ln \mu}{\alpha^{*}} .
$$

Theorem 3.3. For a given constant $\alpha>0, \beta>0$ and time-varying delay satisfying (2.3), suppose that the subsystem $(1 \leq i \leq k)$ of switched system (2.1) satisfies the conditions of Lemma 3.1, and the others satisfy the conditions of Lemma 3.2. Then the system (2.1) is exponentially stable for switching signal satisfying (3.36), (3.37) and the state decay estimate is given by

$$
\|x(t)\| \leq \sqrt{\frac{c_{0} b_{i}}{a_{i}}} e^{-\lambda\left(t-t_{0}\right)}\left\|x\left(t_{0}\right)\right\|_{\mathrm{cl}}
$$

where

$$
\begin{aligned}
c_{0}= & e^{N_{0} \ln \mu}, \quad \quad \quad=\frac{1}{2}\left(\alpha^{*}-\frac{\ln \mu}{T_{a}}\right), \quad \mathcal{L}=\min _{1 \leq i \leq m} \lambda_{\min }\left(P_{i}^{-1}\right), \\
\mathcal{M}= & \max _{1 \leq i \leq m} \lambda_{\max }\left(P_{i}^{-1}\right)+\max _{1 \leq i \leq m} 2 h_{2 i} \lambda_{\max }\left[P_{i}^{-1} Q_{i} P_{i}^{-1}\right]+\max _{1 \leq i \leq m} 2 h_{2 i}^{3} \lambda_{\max }\left[P_{i}^{-1} R_{i} P_{i}^{-1}\right] \\
& +\max _{1 \leq i \leq m} \eta_{i}^{3} \lambda_{\max }\left[P_{i}^{-1} U_{i} P_{i}^{-1}\right]+\max _{1 \leq i \leq m} k_{i}^{2} \lambda_{\max }\left[P_{i}^{-1} S_{i} P_{i}^{-1}\right],
\end{aligned}
$$

and there exists $\mu \geq 1$ such that

$$
P_{i} \leq \mu P_{j}, \quad Q_{i} \leq \mu Q_{j}, \quad R_{i} \leq \mu R_{j}, \quad U_{i} \leq \mu U_{j}, \quad S_{i} \leq \mu S_{j}, \quad \forall i, j \in M .
$$

Proof. Suppose that $t \in\left[t_{k}, t_{k+1}\right)$. For piecewise Lyapunov functional candidate (3.1) and (3.23) along trajectory of network system (2.1), we have

$$
V(x(t)) \leq \begin{cases}e^{-\alpha\left(t-t_{k}\right)} V_{i}\left(x\left(t_{k}\right)\right), & \text { if } i \in 1,2, \ldots, k \\ e^{\beta\left(t-t_{k}\right)} V_{j}\left(x\left(t_{k}\right)\right), & \text { if } j \in k+1, k+2, \ldots, m .\end{cases}
$$


Since $V_{\sigma\left(t_{k}\right)}\left(x\left(t_{k}\right)\right) \leq \mu V_{\sigma\left(t_{k}^{-}\right)}\left(x\left(t_{k}^{-}\right)\right)$is true from (3.34) at the switching point $t_{k}$, where $t_{k}^{-}=\lim _{t \rightarrow t_{k}} t$ and the relation $k=N_{\sigma}\left(t_{0}, t\right) \leq N_{0}+\left(\left(t-t_{0}\right) / T_{a}\right)$, we obtain

$$
\begin{aligned}
V(x(t)) & \leq e^{\beta T^{+}\left(t_{k}, t\right)-\alpha T^{-}\left(t_{k}, t\right)} V_{\sigma\left(t_{k}\right)}\left(x\left(t_{k}\right)\right) \\
& \leq e^{\beta T^{+}\left(t_{k}, t\right)-\alpha T^{-}\left(t_{k}, t\right)} \mu V_{\sigma\left(t_{k}^{-}\right)}\left(x\left(t_{k}^{-}\right)\right) \\
& \leq e^{\beta T^{+}\left(t_{k}, t\right)-\alpha T^{-}\left(t_{k}, t\right)} \mu e^{\beta T^{+}\left(t_{k-1}, t_{k}\right)-\alpha T^{-}\left(t_{k-1}, t_{k}\right)} V_{\sigma\left(t_{k-1}\right)}\left(x\left(t_{k-1}\right)\right) \\
& \leq \mu e^{\beta T^{+}\left(t_{k-1}, t\right)-\alpha T^{-}\left(t_{k-1}, t\right)} V_{i \sigma\left(t_{k-1}\right)}\left(x\left(t_{k-1}\right)\right) \\
& \vdots \\
& \leq \mu^{k} e^{\beta T^{+}\left(t_{0}, t\right)-\alpha T^{-}\left(t_{0}, t\right)} V_{\sigma\left(t_{0}\right)}\left(x\left(t_{0}\right)\right) \\
& \leq e^{\beta T^{+}\left(t_{0}, t\right)-\alpha T^{-}\left(t_{0}, t\right)+\left(N_{0}+\left(\left(t-t_{0}\right) / T_{a}\right)\right) \ln \mu} V_{\sigma\left(t_{0}\right)}\left(x\left(t_{0}\right)\right)
\end{aligned}
$$

Under the switching law (S1) for any $t_{0}, t$, we have

$$
\begin{aligned}
\beta T^{+}\left(t_{0}, t\right)-\alpha T^{-}\left(t_{0}, t\right) & \leq-\alpha^{*}\left(T^{+}\left(t_{0}, t\right)+T^{-}\left(t_{0}, t\right)\right) \\
& =-\alpha^{*}\left(t-t_{0}\right) .
\end{aligned}
$$

Thus,

$$
\begin{aligned}
V(x(t)) & \leq e^{N_{0} \ln \mu} e^{-\left(\alpha^{*}-\left(\ln \mu / T_{a}\right)\right)\left(t-t_{0}\right)} V_{\sigma\left(t_{0}\right)}\left(x\left(t_{0}\right)\right) \\
& \leq c_{0} e^{-2 \lambda\left(t-t_{0}\right)} V_{\sigma\left(t_{0}\right)}\left(x\left(t_{0}\right)\right)
\end{aligned}
$$

where $c_{0}=e^{N_{0} \ln \mu}, \lambda=(1 / 2)\left(\alpha^{*}-\left(\ln \mu / T_{a}\right)\right)$. According to (3.33), we have

$$
\mathcal{L}\|x(t)\|^{2} \leq V(x(t)), \quad V_{\sigma\left(t_{0}\right)}\left(x\left(t_{0}\right)\right) \leq \mathcal{M}\left\|x\left(t_{0}\right)\right\|_{\mathrm{cl}}^{2}
$$

Combining (3.44) and (3.45) leads to

$$
\|x(t)\|^{2} \leq \frac{1}{\rho} V(x(t)) \leq \frac{\mathscr{M}}{\rho} c_{0} e^{-2 \lambda\left(t-t_{0}\right)}\left\|x\left(t_{0}\right)\right\|_{\mathrm{cl}}^{2} .
$$

Therefore,

$$
\|x(t)\| \leq \sqrt{\frac{c_{0} \mathcal{M}}{\mathcal{L}}} e^{-\lambda\left(t-t_{0}\right)}\left\|x\left(t_{0}\right)\right\|_{\mathrm{cl}} .
$$

which means that solution to (2.1) is exponentially stable. The proof is thus completed. 


\section{Numerical Example}

In this section, we now provide an example to show the effectiveness of the result in Theorem 3.3.

Example 4.1. Consider the nonlinear systems with interval time-varying and distributed delays (2.1) with the following parameters:

$$
\begin{aligned}
\dot{x}(t)= & A_{\sigma(t)} x(t)+D_{\sigma(t)} x\left(t-h_{\sigma(t)}(t)\right)+C_{\sigma(t)} \int_{t-k_{\sigma(t)}(t)}^{t} x(s) d s \\
& +E_{\sigma(t)} f_{\sigma(t)}\left(t, x(t), x\left(t-h_{\sigma(t)}(t)\right), \int_{t-k_{\sigma(t)}(t)}^{t} x(s) d s\right),
\end{aligned}
$$

where

$$
\begin{gathered}
A_{1}=\left[\begin{array}{cc}
-2 & 0.1 \\
0 & -1
\end{array}\right], \quad D_{1}=\left[\begin{array}{cc}
0.1 & 0 \\
0.3 & -0.5
\end{array}\right], \quad C_{1}=\left[\begin{array}{cc}
0.1 & 2 \\
0 & 0.2
\end{array}\right], \quad E_{1}=\left[\begin{array}{cc}
0.1 & 0 \\
0 & 0.1
\end{array}\right], \\
A_{2}=\left[\begin{array}{cc}
-1 & 0.1 \\
0.1 & 0.1
\end{array}\right], \quad D_{2}=\left[\begin{array}{cc}
0.2 & 0 \\
0.1 & -1.3
\end{array}\right], \quad C_{2}=\left[\begin{array}{cc}
0.25 & 0.2 \\
0 & 0.1
\end{array}\right], \quad E_{2}=\left[\begin{array}{cc}
0.1 & 0 \\
0 & 0.1
\end{array}\right], \\
\begin{aligned}
f_{1}\left(t, x_{1}(t), x_{1}(t-h(t)), \int_{t-k_{1}(t)}^{t} x_{1}(s) d s\right) \leq & 0.1\left\|x_{1}(t)\right\|+0.1\left\|x_{1}(t-h(t))\right\| \\
& +0.2\left\|\int_{t-k_{1}(t)}^{t} x_{1}(s) d s\right\|, \\
f_{2}\left(t, x_{2}(t), x_{2}(t-h(t)), \int_{t-k_{2}(t)}^{t} x_{2}(s) d s\right) \leq & 0.1\left\|x_{2}(t)\right\|+0.05\left\|x_{2}(t-h(t))\right\| \\
& +0.1\left\|\int_{t-k_{2}(t)}^{t} x_{2}(s) d s\right\| .
\end{aligned}
\end{gathered}
$$

Given $h_{11}=0.1, h_{12}=0.2, h_{21}=0.15, h_{22}=0.25, k_{1}=0.22, k_{2}=0.2, \alpha=0.25$, and $\beta=0.2$, it is found that LMIs (3.3), (3.4), (3.5), (3.25), (3.26), and (3.27) of Lemmas 3.1 and 3.2 have feasible solutions. Thus, we can get $\mu=1.35$. We now choose $\alpha^{*}=0.155<\alpha$. Then, the switching law (S1) will require $\inf _{t \geq t_{0}} T^{-}\left(t_{0}, t\right) / T^{+}\left(t_{0}, t\right) \geq\left(\left(\beta+\alpha^{*}\right) /\left(\alpha-\alpha^{*}\right)\right)=4.2632$ and the average dwell time is computed as $T_{a} \geq T_{a}^{*}=\ln \mu / \alpha^{*}=3.6472$.

We let $h_{1}(t)=0.1+0.05|\cos t|, k_{1}(t)=0.1|\sin t|, h_{2}(t)=0.1+0.05|\sin t|, k_{2}(t)=0.1|\cos t|$ and $\phi(t)=[-\cos t, \cos t]$, for all $t \in[-0.25,0]$. Figure 1 shows the trajectories of solutions $x_{1}(t)$ and $x_{2}(t)$ of the interval time-varying and distributed delays system.

\section{Conclusions}

In this paper, we have investigated the exponential stability for a class of switched nonlinear systems with discrete and distributed time-varying delays. The interval time-varying delay 


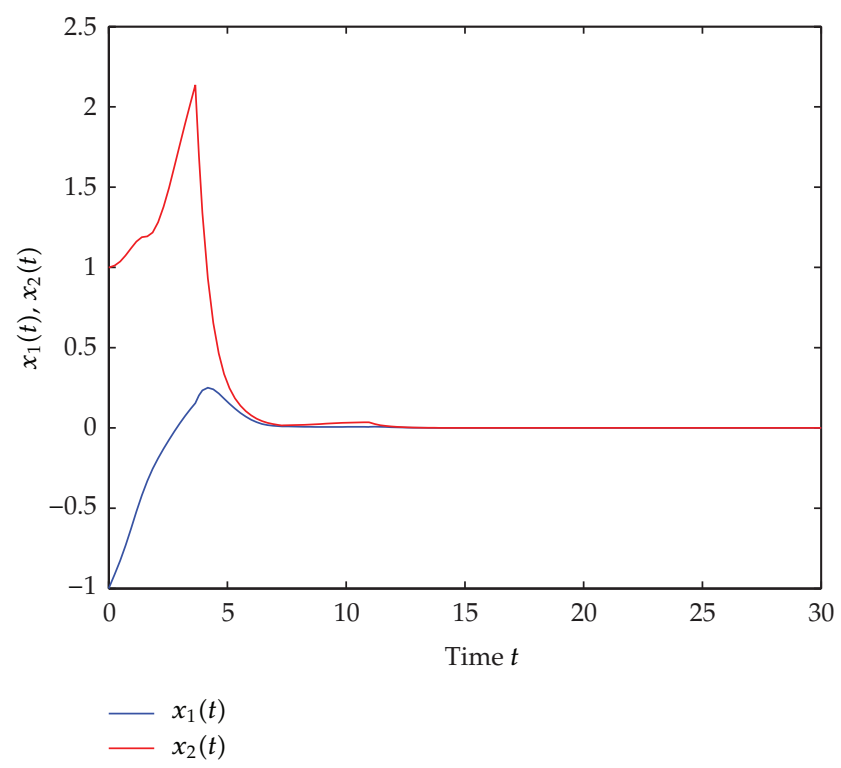

Figure 1: The trajectories of $x_{1}(t)$ and $x_{2}(t)$ of the interval time-varying delay system (4.1).

function is not necessary to be differentiable which allows time-delay function to be a fast time-varying function. By using the average dwell-time approached and improved piecewise Lyapunov-Krasovskii functionals which are constructed based on and combined with Leibniz-Newton's formula, for the cases that subsystems are stable and unstable, some new delay-dependent stability criteria are derived by a set of linear matrix inequalities. Numerical examples are given to illustrate the effectiveness of our theoretical results.

\section{Acknowledgments}

The first author is supported by the Graduate School, Chiang Mai University and the Centre of Excellence in Mathematics, the Commission on Higher Education, Thailand. The second and the third authors are supported by the Centre of Excellence in Mathematics, the Commission on Higher Education, Thailand.

\section{References}

[1] S. Engell, S. Kowalewski, C. Schulz, and O. Stursberg, "Continuous-discrete interactions in chemical processing plants," Proceedings of the IEEE, vol. 88, no. 7, pp. 1050-1068, 2000.

[2] R. Horowitz and P. Varaiya, "Control design of an automated highway system," Proceedings of the IEEE, vol. 88, no. 7, pp. 913-925, 2000.

[3] C. Livadas, J. Lygeros, and N. A. Lynch, "High-level modeling and analysis of the traffic alert and collision avoidance system (TCAS)," Proceedings of the IEEE, vol. 88, no. 7, pp. 926-948, 2000.

[4] P. Varaiya, "Smart cars on smart roads. Problems of control," IEEE Transactions on Automatic Control, vol. 38, no. 2, pp. 195-207, 1993.

[5] D. Pepyne and C. Cassandaras, "Optimal control of hybrid systems in manufacturing," Proceedings of the IEEE, vol. 88, pp. 1008-1022, 2000.

[6] M. Song and T. J. Tarn, "Integration of task scheduling, action planning, and control in robotic manufacturing systems," Proceedings of the IEEE, vol. 88, no. 7, pp. 1097-1107, 2000. 
[7] D. Liberzon and A. S. Morse, "Basic problems in stability and design of switched systems," IEEE Control Systems Magazine, vol. 19, no. 5, pp. 59-70, 1999.

[8] X. M. Sun, D. Wang, W. Wang, and G. H. Yang, "Stability analysis and L2-gain of switched delay systems with stable and unstable subsystems," in Proceedings of the IEEE 22nd International Symposium on Intelligent Control (ISIC '07), pp. 208-213, October 2007.

[9] G. Zhai, B. Hu, K. Yasuda, and A. N. Michel, "Stability analysis of switched systems with stable and unstable subsystems: an average dwell time approach," International Journal of Systems Science, vol. 32, no. 8, pp. 1055-1061, 2001.

[10] J. P. Hespanha and A. S. Morse, "Stability of switched systems with average dwell-time," in Proceedings of the 38th IEEE Conference on Decision and Control (CDC '99), pp. 2655-2660, December 1999.

[11] G. Zhai, B. Hu, K. Yasuda, and A. N. Michel, "Piecewise lyapunov functions for switched systems with average dwell time," Asian Journal of Control, vol. 2, no. 3, pp. 192-197, 2000.

[12] J. H. Park and O. Kwon, "Novel stability criterion of time delay systems with nonlinear uncertainties," Applied Mathematics Letters, vol. 18, no. 6, pp. 683-688, 2005.

[13] J. H. Park and H. Y. Jung, "On the exponential stability of a class of nonlinear systems including delayed perturbations," Journal of Computational and Applied Mathematics, vol. 159, no. 2, pp. 467-471, 2003.

[14] Q. L. Han, "Robust stability for a class of linear systems with time-varying delay and nonlinear perturbations," Computers and Mathematics with Applications, vol. 47, no. 8-9, pp. 1201-1209, 2004.

[15] H. R. Karimi, M. Zapateiro, and N. Luo, "New delay-dependent stability criteria for uncertain neutral systems with mixed time-varying delays and nonlinear perturbations," Mathematical Problems in Engineering, vol. 2009, Article ID 759248, 22 pages, 2009.

[16] Y. Chen, A. Xue, R. Lu, and S. Zhou, “On robustly exponential stability of uncertain neutral systems with time-varying delays and nonlinear perturbations," Nonlinear Analysis: Theory, Methods and Applications, vol. 68, no. 8, pp. 2464-2470, 2008.

[17] V. L. Kharitonov and D. Hinrichsen, "Exponential estimates for time delay systems," Systems and Control Letters, vol. 53, no. 5, pp. 395-405, 2004.

[18] V. B. Kolmanovskii, S. I. Niculescu, and J. P. Richard, "On the Liapunov-Krasovskii functionals for stability analysis of linear delay systems," International Journal of Control, vol. 72, no. 4, pp. 374-384, 1999.

[19] V. N. Phat and A. V. Savkin, "Robust state estimation for a class of uncertain time-delay systems," Systems and Control Letters, vol. 47, no. 3, pp. 237-245, 2002.

[20] V. N. Phat and P. T. Nam, "Exponential stability criteria of linear non-autonomous systems with multiple delays," Electronic Journal of Differential Equations, vol. 58, pp. 1-9, 2005.

[21] T. Botmart and P. Niamsup, "Robust exponential stability and stabilizability of linear parameter dependent systems with delays," Applied Mathematics and Computation, vol. 217, no. 6, pp. 2551-2566, 2010.

[22] I. Amri, D. Soudani, and M. Benrejeb, “Exponential stability and stabilization of linear systems with time varying delays," in Proceedings of the 6th International Multi-Conference on Systems, Signals and Devices (SSD '09), March 2009.

[23] L. V. Hien and V. N. Phat, "Exponential stability and stabilization of a class of uncertain linear timedelay systems," Journal of the Franklin Institute, vol. 346, no. 6, pp. 611-625, 2009.

[24] Q. L. Han, "A descriptor system approach to robust stability of uncertain neutral systems with discrete and distributed delays," Automatica, vol. 40, no. 10, pp. 1791-1796, 2004.

[25] X. G. Li and X. J. Zhu, "Stability analysis of neutral systems with distributed delays," Automatica, vol. 44, no. 8, pp. 2197-2201, 2008.

[26] T. Li, Q. Luo, C. Sun, and B. Zhang, "Exponential stability of recurrent neural networks with timevarying discrete and distributed delays," Nonlinear Analysis: Real World Applications, vol. 10, no. 4, pp. 2581-2589, 2009.

[27] F. Gao, S. Zhong, and X. Gao, "Delay-dependent stability of a type of linear switching systems with discrete and distributed time delays," Applied Mathematics and Computation, vol. 196, no. 1, pp. 24-39, 2008.

[28] K. Gu, V. L. Kharitonov, and J. Chen, Stability of Time-Delay System, Birkhauser, Boston, Mass, USA, 2003. 


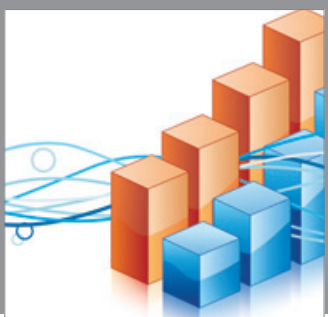

Advances in

Operations Research

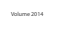

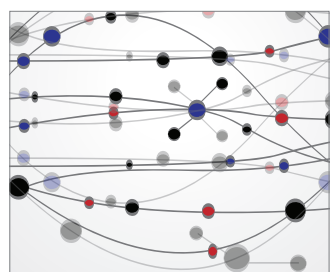

\section{The Scientific} World Journal
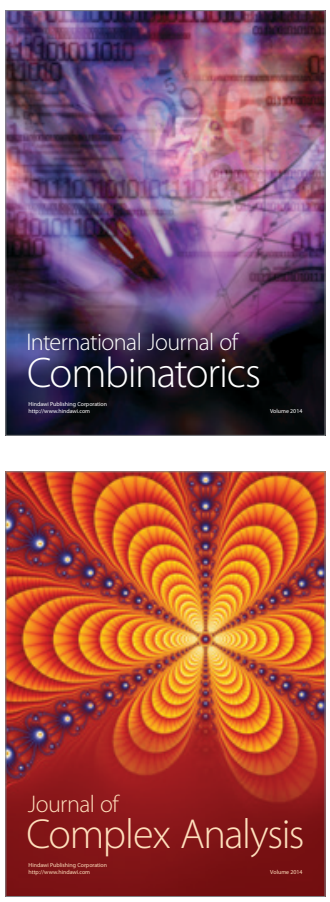

International Journal of

Mathematics and

Mathematical

Sciences
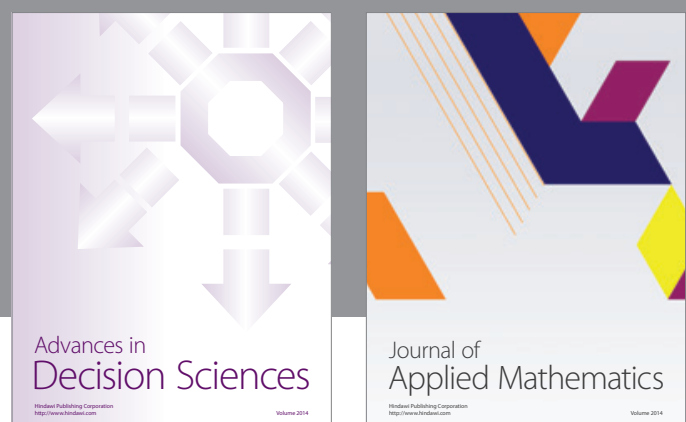

Journal of

Applied Mathematics
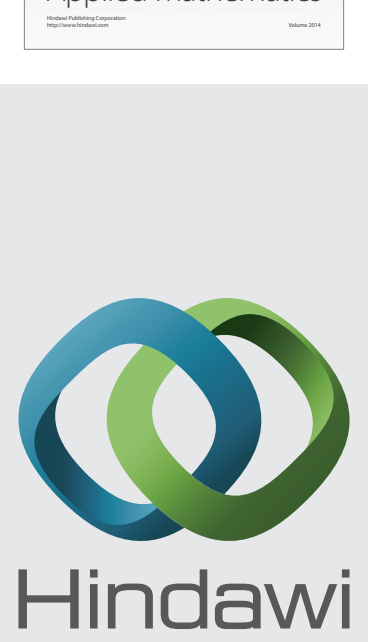

Submit your manuscripts at http://www.hindawi.com
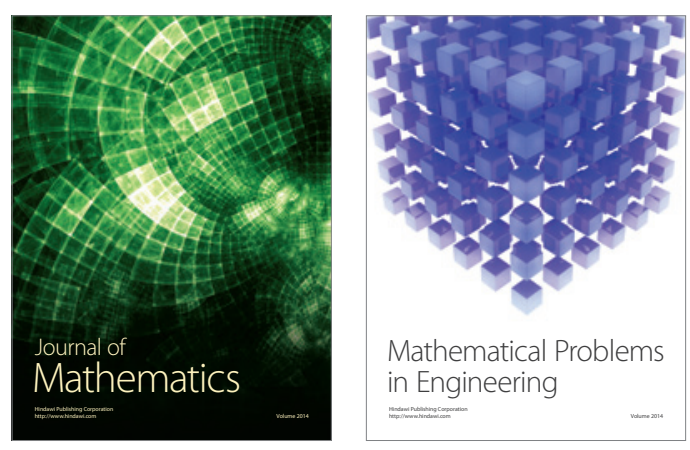

Mathematical Problems in Engineering
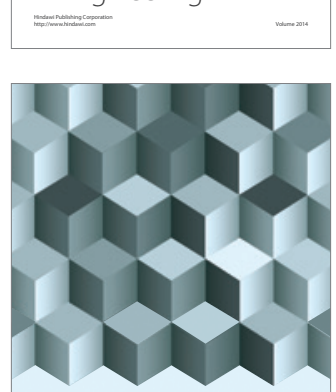

Journal of

Function Spaces
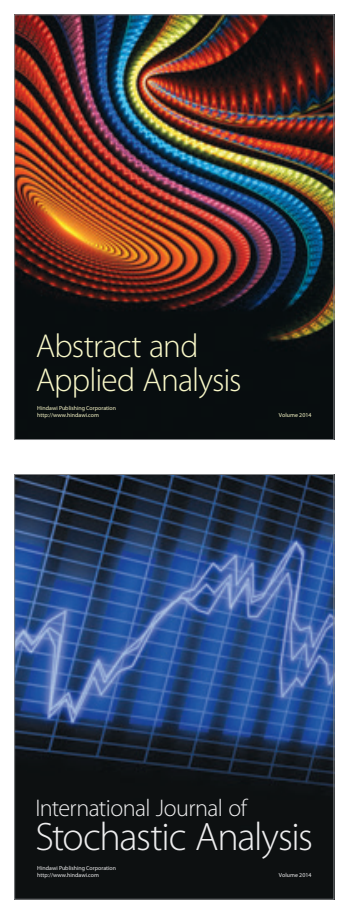

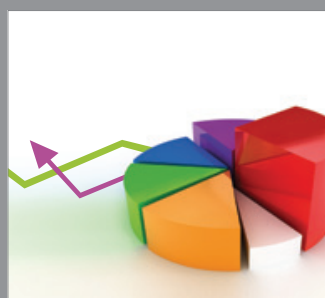

ournal of

Probability and Statistics

Promensencen
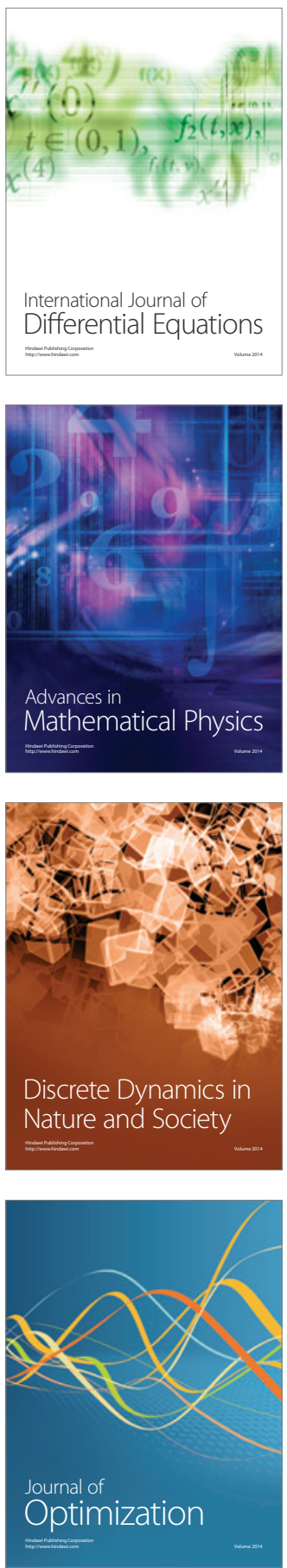